\title{
A BAYESIAN APPROACH TO EXTRACTING MEANING FROM SYSTEM
} BEHAVIOR*

$$
\text { CONF-9810/9-- }
$$

William B. Dress

Oak Ridge National Laboratory

P.O. Box 2008

Oak Ridge, Tennessee 37831-6011

(423) $574-4801$
RECEIVED

JUL 291998

OSTI

To be presented at the

1998 IEEE International Conference on Systems, Man, and Cybernetics

La Jolla, California

October 11-14, 1998

"The submitted manuscript has been authored by a contractor of the U.S.

Govenment under contract No. DE-AC05-96OR22464. Accordingly, the

U.S. Government retains a nonexclusive, royalty-free license to publish or

reproduce the published form of this contribution, or allow others to do so,

for U.S. Govemment purposes."

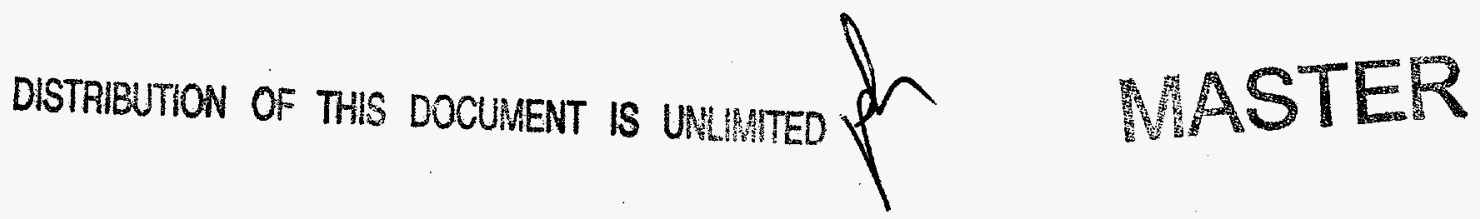

"This research was performed at OAK RIDGE NATIONAL LABORATORY, managed by LOCKHEED MARTIN ENERGY RESEARCH CORP. for the U.S. DEPARTMENT OF ENERGY under contract DE-AC05-960R22464. 


\section{DISCLAIMER}

This report was prepared as an account of work sponsored by an agency of the United States Government. Neither the United States Government nor any agency thereof, nor any of their employees, makes any warranty, express or implied, or assumes any legal liability or responsibility for the accuracy, completeness, or usefulness of any information, apparatus, product, or process disclosed, or represents that its use would not infringe privately owned rights. Reference herein to any specific commercial product, process, or service by trade name, trademark, manufacturer, or otherwise does not necessarily constitute or imply its endorsement, recommendation, or favoring by the United States Government or any agency thereof. The views and opinions of authors expressed herein do not necessarily state or refiect those of the United States Government or any agency thereof. 


\section{DISCLAIMER}

Portions of this document may be illegible in electronic image products. Images are produced from the best available original document. 


\title{
A Bayesian approach to extracting meaning from system behavior
}

\author{
William Dress \\ Instrumentation and Controls Division \\ Oak Ridge National Laboratory \\ PO Box 2008 MS 6011 \\ Oak Ridge, Tennessee 37831-6011
}

\begin{abstract}
The modeling relation and its reformulation to include the semiotic hierarchy is essential for the understanding, control, and successful re-creation of natural systems. This presentation will argue for a careful application of Rosen's modeling relationship to the problems of intelligence and autonomy in natural and artificial systems. To this end, I discuss the essential need for a correct theory of induction, learning, and probability; and suggest that modern Bayesian probability theory, developed by Cox, Jaynes, and others, can adequately meet such demands, especially on the operational level of extracting meaning from observations.
\end{abstract}

The methods of Bayesian and Maximum Entropy parameter estimation have been applied to measurements of system observables to directly infer the underlying differential equations generating system behavior. This approach bypasses the usual method of parameter estimation based on assuming a functional form for the observable and then estimating the parameters that would lead to the particular observed behavior. The computational savings is great since only location parameters enter into the maximum-entropy calculations; this innovation finesses the need for nonlinear parameters altogether. Such an approach more directly extracts the semantics inherent in a given system by going to the root of system meaning as expressed by abstract form or shape, rather than in syntactic particulars, such as signal amplitude and phase. Examples will be shown how the form of a system can be followed while ignoring unnecessary details. In this sense, we are observing the meaning of the "words" rather than being concerned with their particular expression or "language." For the present discussion, empirical models are embodied by the differential equations underlying, producing, or describing the behavior of a process as measured or tracked by a particular variable setthe observables. The a priori models are probability structures that capture syntactical relationships within the formal system that mirrors the natural system under observation. Inductive learning is then a prescription for incorporating the current, and possibly changing, empirical model into an iterative syntactical relationship in the form of a statement about parameters, producing an updated a priori model subject to future modification in the light of changing parameter sets.

The probabilistic nature of the model descriptions replaces the rigid structures normally used with a flexible descriptions. These structures then evolve both with new knowledge about the system under study as well as with temporal evolution as the system changes its form or structure in response to environmental interactions or internal dynamics. The implications for anticipatory control and understanding of natural and artificial systems are evident and unfold into possibilities for the creation of complex artificial systems.

\section{INTRODUCTION}

The purpose of this introduction is to suggest a framework that, while retaining the structure and function of semiosis, follows a clearer path, at least to my mind, from observation to modeling to (perhaps) artifact construction. After exploring semiotics for a short distance, the remainder of the introduction will present an attempt to synthesize a framework capable of accommodating both the science and engineering of intelligent systems in a way that retains structures analogous to, if not identical with, semiosis as defined by Peirce and elaborated by others. To this end, more detail of the applied semiotics program will be presented.

Then I introduce Popper's three worlds as the general framework and Rosen's theory of the modeling relation to contain the specific structure for exploring intelligent systems. It will then be a simple identification of concepts and relationships to show how semiosis maps into Rosen's clear and specific ideas of the modeling relation. It will become clear that the essential structure of semiosis is not only present in the modeling relation, but the latter is more appropriate for analytic and constructive purposes.

The rest of the paper presents a discussion of how some particular models might be abstracted from the natural world and perhaps be reintroduced into the natural world as artifacts. The modeling relation provides a formalism to illustrate certain pragmatic methods of system identification and to extract meaning from system behavior.

\section{Semiotics and Applied Semiosis}

The semiotic triad of Peirce is seen as an indispensable framework for analyzing natural language into what is being discussed, the signs used in a discussion, and the meaning inherent in the discussion. This "unresolvable" triad of \{object, sign, interpretant\} central to semiosis, is defined by Peirce as

[A]n action, or influence, which is, or involves, an operation of three subjects, such as a sign, its object and its interpretant, this tri-relative influence not being in any way resolvable into an action between pairs. - C.

\section{S. Peirce, quoted by Eco $[1$, p. 1$]$}

This seminal formulation of semiosis can be interpreted as containing the promise of a framework that will contribute to, if not solve, human-machine and machine-machine communication problems. For this reason, there has been much recent excitement in exploring semiotics for such purposes as machine intelligence and the control of intelligent artifacts. This "applied semiotics" seems to have made important progress in the area of the creation and control of intelligent machines. An outstanding example of such progress is demonstrated by the Meystel-Albus collaboration at The National Institute of Standards and Technology (NIST) and may be examined in several recent publications $[2,3,4]$ and a 
symposium, "Intelligent Systems: A Semiotic Perspective," held for the past three years at NIST. In contrast to this applied semiotics, the main body of literature in the field concerns linguistic-philosophical endeavors as exemplified by the works Thomas Sebeok (as a charming example, see [5]), Umberto Eco (for example, [1]), and many others (see [6] for some of the breadth of this field of knowledge). Indeed, Eco makes a strong case that the semiotic triad is an abstraction designed for the purely philosophical exposition of the general theory of signs (semiotics) as it pertains to natural language and philosophical thought. After discussing the role of philosophy in constructing world views and its necessary limitations as a predictive mechanism, Eco [1, p. 12] states explicitly that "a general semiotics studies the whole of the human signifying activity-languages-and languages are what constitutes human beings..." This declaration is pregnant with possibilities, but seems too expansive and difficult a first step for a systems theory of machine intelligence when there are pressing needs for applications. Since inferences about systems and their actions-predictions, in other words - are required for the design and control of intelligent systems, the implication of Eco's caution seems to be that an applied semiosis is premature without an additional philosophical-theoretical undertaking that would lead to the assurance opredictive capability. That is, we first must develop a theory of semiosis so it can capture the full range of scientific activity-from description, through inference, to prediction. Since prosaic activities of engineering and construction of artifacts are of interest here, it is perhaps a better course to seek a framework more suited to doing science and engineering rather than attempt to extend what is an essentially philosophical discipline. On the other hand, the abstract idea of the Peircean triad tempts one to assert, in incautious moments, that semiotics may be essential for the process of communication with and within intelligent machines. Below, I show how to retain the essential features of the semiotic process for system modeling and control by reordering the concepts with slightly different emphasis and perhaps an alternate nomenclature by embedding those concepts into a larger framework and then turning to a specific relationship between natural or material systems and formal models thereof.

Meystel's "functional diagram" of semiosis [2] is an augmentation of the Peircean semiotic triad of object-signinterpretant with the identifications of "syntax" for "sign", "semantics" for "interpretant", and "pragmatics" for "object" wherein the processes of transduction, representation, and interpretation/application connect the three nodes as shown in the implied triangle

$\rightarrow$ Pragmatics-TRANSDUCE $\rightarrow$ Syntax $\rightarrow$ REPRESENT $\rightarrow$ Semantics - APPLY $\rightarrow$

where the initial and final arrows are congruent. Viewed as semiosis, this is a purely descriptive framework for analyzing the linguistic structure of natural language. Meystel has given it an operational interpretation by selecting certain transformations in systems and control theory that he identifies with parts of the above structure. Indeed, an elaborate and rich version of systems control can be constructed based on these ideas.

Popper's Three Worlds

Karl Popper, who referred to C. S. Peirce as "the great American mathematician and physicist, and I believe, one of the greatest philosophers of all time" [7, p. 251], introduced the three-worlds concept in an essay on the difference between subjective and objective knowledge in the following passage.
[W]ithout taking the words 'world' or 'universe' too seriously, we may distinguish the following three worlds or universes: first, the world of physical objects or physical states; secondly, the world of states or consciousness, or of mental states, or perhaps of behavioral dispositions to act; and thirdly, the world of objective contents of thought, especially of scientific and poetic thoughts and works of art.-Karl Popper, [7, p. 59]

Popper then goes on to make a strong case for the objective existence of these three worlds. The three-world concept is an obvious extension of Plato's idealistic world of forms, yet is operationally convenient for many discussions. In what follows, I require the three-worlds concept as a framework for discourse; conjectures as to its objective nature, while interesting, are not necessary here.

The "objects" of the Peircean triad clearly belong to world 1 - the world of things that exist independently of thought and culture. The "signs" inhabit world 3-the world of ideas and theories as embodied in material world-1 objects such as books and libraries. Of course, physical signs are objects and thence belong to world 1 ; but that is a trivial and amusing identification much as Lewis Carroll's logical play with "the name of the name is called...," which is the most concise statement of semiosis possible. World 2 is the world of mental activities and states-underlying perception so necessary for creating corresponding world-3 entities. The "interpretants" or semantics appear as relationships between world 2 and world 3 reflecting back onto world 1 -indeed it is shown below that this is consistent with Peirce's view of semantics. The connectives of transducing, representing, and applying in the augmented semiotic triad are of course processes and, when carried out by human beings, necessarily involve an interaction between world 1 and world 3, mediated by world- 2 entities. When, and if, this interaction is carried out by intelligent constructs, the very idea of world 3 may have to change. The three-world concept resonates nicely with the Peircean triad and the fundamental idea of semiotics by accommodating the semiotic triad as well as the processes deemed necessary for applied semiotics. It also serves as a framework for the modeling-relation triad of natural system, encoding and decoding, and formal system as shown in the next section.

\section{Rosen's Modeling Relation}

Following Rosen $[8,9]$, consider a natural or material system, $\mathcal{N}$, and a formal system, $\mathcal{F}$. The natural system consists of components and perhaps specifiable entities, but any identification leads us into the formal system. As with all natural systems that are interesting, let $N$ exhibit behaviors that can be observed by the process of measurement. The temporal evolution taking place in such an $\mathcal{N}$ is an internal entailment that is usually called causality. $\mathcal{F}$ likewise has components in the form of elements, perhaps axioms or theorems, and possesses an entailment structure in the form of production rules that may be identified with inference. This entailment process is an internal operation within $\mathcal{F}$. So far, the description is that of two isolated entities

$\mathcal{N}$ (components, causality) and $\mathcal{F}$ (elements, implication) The measurement process is subsumed by the concept of encoding behaviors of $\mathcal{N}$ into elements or perhaps sets in $\mathcal{F}$. Inferences can now be made in $\mathcal{F}$ by "turning" the mathematical "crank." The results of the inferences must then be compared to the future behavior of $\mathcal{N}$ a process called "prediction" in science. This decoding is a dual process to encoding - one that the scientist uses to verify predictions by another measurement process on the future behavior of $\mathcal{N}$. This leads to the idea of connectives, or a relation, between 
$\mathcal{N}$ and $\mathcal{F}$ that allow comparison of the two systems; thus the description is now

$$
\mathcal{N}(\text { components, causality }) \rightleftharpoons \mathcal{F} \text { (elements, implication })
$$

where the connective " $\rightleftharpoons$ " stands for both encoding and decoding-processes that stand outside the causal structure in $\mathcal{N}$ and formal entailment in $\mathcal{F}$. Indeed, " $\rightleftharpoons$ " is where the creative aspects of science and engineering are needed most.

$\mathcal{N}$ and its components clearly belong to world 1 and the encodings and decodings as processes belong to world 2 while being conceptualized, and thence to world 3 , as formal procedures. $\mathcal{F}$ is clearly a world 3 construct, while the inferences are world-2 activities or world-3 implications depending on who or what is performing them. Implications in the world-1 system-that is, causality-remain strictly a world-1 activity or relation.

\section{Formal Model in System Identification}

Measurement, abstraction, and transduction are all instances of the connective "Transduce" in applied semiosis. When comparing formal systems, the encoding concept is best thought of as a process of abstraction. Comparisons between natural and formal systems require the encoding process of measurement. The term transduction is a generic name for measurement and can subsume abstraction as well. Of course, common usage also places transduction on the decoding side as well, allowing for a certain amount of confusion when entailment processes in different systems are to be compared. This framework allows the comparison of material and formal systems in a clear and unambiguous manner. The process of hypothesis testing, used below to construct formal models from observed behaviors, is nothing but an iterative and comparative application of a set of formal models $\left\{\mathcal{F}_{k}\right\}$ to a single natural system. As such, it nicely captures the activity of system identification and parameter estimation, and allows extension of these ideas to artificial constructs as embodiments of material (i.e., natural) systems that capture specific identified behaviors. Another compelling reason for embedding discussions of intelligent and autonomous systems in Rosen's development of the modeling relation is that he has shown $[9$, p. $117 \mathrm{ff}$.] that the modeling framework is not only powerful enough to contain science in the form of Newtonian states (i.e., classical as well as quantum theories), but can also entertain the concept of organism, presently not accessible to reductionist programs.

Meaning Based on Entailment-Inference and Causality As above, consider a natural or material system $\mathcal{N}$, a formal system $\mathcal{F}$, and a relation between them specified by a particular set of encodings and decodings. If entailment or implication in $\mathcal{F}$ is verified, upon decoding, as a prediction of entailment or causality in $\mathcal{N}$, the model is valid or good in some sense. Whether it captures all the entailments in $\mathcal{N}$ that we are interested in is another question. The diagram for the modeling relation is

$$
\mathbb{N} \rightleftharpoons \mathcal{F} \sharp
$$

where $\sharp$ represents the entailment process within either system and $\rightleftharpoons$ represents the external encoding and decoding processes. When reading expositions of formal systems, which by definition are syntactical, the phrase "semantics" often appears. Usually, as in Pospelov's definition of a formal system [10], this term is taken as a special inference within the formal system that demonstrates correspondence between two elements of the formal system. In other words, "semantics" is really syntactical inference within a formal system. While a well-defined mathematical (i.e., formal) op- eration, it really has no meaning when seeking to establish comparisons between formal systems and natural systems with the goal of generating other natural systems with specific properties peculiar to biological organisms. That is, all usages of "meaning" within purely formal systems can be shown to be isomorphic to syntactical inferences within a formal system, perhaps augmented by additional symbols that connote similarity or identity of elements or sets.

Since formal embeddings of semantics, perhaps interesting in their own right, are non-productive for the present discussion, it becomes reasonable to pursue the meaning of meaning for a while. "Meaning" or "semantics" presupposes the ability to ask "why?" This is impossible in formal systems as any scientist knows-science can only answer "how" questions, since "why" questions refer to a final cause in Aristotle's nomenclature. Final causes have been studiously avoided in science and exorcised when found (e.g., vitalism and all forms of teleos). For example, "Why did the organism do that?" can only mean "How did the organism behave in response to certain inputs?"-any hint of intentionality is avoided, resulting in behaviorism at its purest. Peirce [11, p. 152] suggests that meaning can only be obtained by referring the abstract (objects in $\mathcal{F}$ ) to the concrete (objects in some $\mathcal{N}$ ) and not the other way. Meaning and semantics then depend on the decoding relation and are intimately concerned with the comparison of entailment mechanisms of the two systems. Of course, by replacing $N$ with another formal system, $\mathcal{F}$, say, we can presume to demonstrate a semantics between formal systems. This may or may not be fruitful, yet is clearly possible. Note that in this case, the meaning still enters from outside the two systems, not within them. Thus, the semantic node in the semiotic triad will be taken as a relation between entailments in comparing two systems--semantics has a foot in both world 1 and world 3 via creative and mental activities of world 2. This is not inconsistent with Peirce's notions and allows us to talk about semantics-to ask "why" regarding organisms and constructs-without violating the great taboo.

In this paper, there is no need to push the semantics to asking the why question, but it is nice to know that it may be possible. Much activity in present-day artificial intelligence, as may be verified by visiting numerous web sites, has rightfully ignored the proscriptions against the appearance of teleos and seems to be comfortable with questions of intentionality that are inherent in such concepts as intelligence, organism, and consciousness. In the remainder of the paper, I will be concerned with procedural ideas of how one might go about capturing behavior in a formal model, extending the model, and perhaps constructing an artifact that goes beyond mere simulation.

\section{EXTRACTING MEANING FROM BEHAVIOR}

Consider the observed dynamics of some variable of a natural system. This dynamics, viewed as a temporal chronicle, is entailed by the system and its interactions with the environment. There is no a priori way to select a model for these dynamics; the system behavior may be described as linear or nonlinear, stochastic or deterministic - such a description is itself a form of model selection. Also, there are many analytic approaches to selecting a "correct" model, ranging from linear systems theory, to deterministic chaos, to methods involving cumulants and higher-order spectra as well as methods seeking to capture and simulate system behavior by using unstructured map simulators such as artificial neural networks. The inventiveness of man has produced a vast arsenal of complicated tools to attack to these issues. To 
compound the problem, without a viable theory of organisms, there is no way of knowing which variable to observe as being the one most effective in establishing the best model. Some have approached this class of problems by setting out elaborate theories of brain, organism, and presupposed system behavior; others by directly analyzing data sets, with little rationale for their particular choice of method other than it has worked in the past on, perhaps, toy problems. (The two à la mode methods being "neural networks" and "chaos.") There seems to be little effort in coordinating methods and theories to obtain the most comprehensive and effective models.

The development followed in the remainder of the paper is to suggest a means of mapping the behavior of a natural or material system onto the structure of a formal system. This is seen as a first step towards consolidating various techniques and a way to aid in theory construction so the correct questions may be asked in the future. I certainly don't have the answers and am merely suggesting that others consider the methods presented here. In particular, I would like to be able to identify a differential equation of a dynamical system as that which "determines" the behavior of the system under observation. This semantics involves an assumption that a differential equation can adequately describe the behavior of an arbitrary natural system. The idea is consistent with the above notion of semantics or meaning associated with the relationship between entailment processes in the natural system under observation and the formal system containing the differential equation. If the semantics of a natural system can indeed be identified this way, perhaps it can also be built into an appropriately constructed artifact.

\section{Bayesian System Identification}

In 1946, Cox [12] demonstrated that any possible form of probabilistic reasoning, that is reasoning under uncertainty or lack of knowledge, is either equivalent to Bayesian inference or is inconsistent. In particular, he showed that the sum and product rules of conditional probability theory are derivable from common sense and consistency considerations. Of course, the product rule on $p(A B \mid C)$-read as "the probability that both hypotheses $A$ and $B$ are true given that $C$ is true"-is simply

$$
p(A B \mid C)=p(A \mid C) p(B \mid A C)=p(B \mid C) p(A \mid B C)
$$

where the latter equality is obtained by the fact that $\mathrm{AB}=\mathrm{BA}$ - the conjunction operation is commutative. Simply solving this relation for $p(A \mid B C)$ results in Laplace's form of Bayes' rule. Let $\mathrm{A}$ be the hypothesis specified by the model, $\mathcal{M}$; $\mathrm{B}$ be the measured or encoded data, $D$; and $\mathrm{C}$ be the background information, $\mathcal{I}$. Then Bayes' rule or theorem is written in the suggestive form

$$
\mathrm{p}(\mathcal{M} \mid \mathcal{D} I)=\mathrm{p}(\mathcal{M} \mid \mathcal{I}) \mathrm{p}(\mathcal{D} \mid \mathcal{M} \mathcal{I}) / \mathrm{p}(\mathcal{D} \mid \mathcal{I}) \text {. }
$$

In words, this expression is usually read as "the posterior probability of the model given the data and the background information is equal to the prior probability of the model times the direct probability of the data normalized by the prior probability, or evidence, of the data." The virtue of Bayes' rule is that it allows an inductive chain to be constructed, with the probability of a particular hypothesis monotonically increasing as new evidence is incorporated. Thus, learning can be thought of as induction based on Bayes' rule, as discussed by Jaynes [13].

The key step in starting the inference implied by Bayes' rule is to assign a numerical value to $\mathrm{p}(\mathcal{D} \mid \mathcal{M} I)$ - the "direct probability" or likelihood of the data. Jaynes [14] accomplished this in 1957 by forming a synthesis of the seemingly unrelated disciplines of information theory and statis- tical mechanics, showing that the principle of maximum entropy can be used as a tool for statistical inference to partially determine probability distributions on the basis of incomplete knowledge. The resulting probability distribution can be used in Bayes' rule to initiate the inference mechanism. Using the method of Lagrange multipliers to maximize the Shannon entropy, it is usually a simple manner to derive the probability for this uncertainty or noise. "Noise" for a Bayesian is simply anything in the set of observations that is not explained by the hypothesis. If there is "too much" noise, or too much variance left unexplained, in the usual statistical language, the hypothesis is probably not very good. Bayesian methods also provide a numerical measure of this quality "probably not very good."

When given a set of models, each one is an hypothesis about the system producing the observed data. Maximum entropy and Bayes' rule may be used to assign each of the hypotheses a likelihood or probability, giving a means of deciding between hypotheses.

\section{Hypotheses as Differential Equations}

Bretthorst [15] emphasized that the Bayesian approach to parameter estimation lets one concentrate on parameters of interest, neglecting the uninteresting ones by repeated applications of the sum rule of probability theory and Bayes' theorem. Other approaches require the solution to a highdimensional problem if there are many such parameters. Replacing the functional-form model directly by a differential equation model accomplishes the same result without the nuisance parameters appearing at all and the nonlinear parameters, such as frequency, become linear ones. Since it is the latter parameters that embody the form of the differential equation, they are the ones necessary for extracting meaning from behavior.

An Example. Suppose that one of the models being considered is

$$
\mathcal{M}(\omega, A, B) \equiv[\mathbf{f}(\mathbf{t})=A \cos \omega t+B \sin \omega t],
$$

and the frequency is of primary interest, the amplitudes being only of marginal interest. Bretthorst [15], following Jaynes [16], showed how one can fit a data set to this model in a maximally noncommittal way by treating the amplitudes as nuisance parameters. This is done by computing the direct probability of the data set that we wish to model. The result is an exponential function of the model parameters and the data. To remove the linear parameters from further consideration, this function is integrated over all possible values of A and B. Bayes' theorem requires knowledge of any prior information concerning these amplitudes. A maximally noncommittal approach is to take $-\infty<\mathrm{A}, \mathrm{B}<+\infty$; if the integrals converge - which they do in this case, then the use of such "improper" or unnormalizable prior densities creates no problems. Bretthorst [15] discusses these issues at length. The resulting probability function has a maximum at the most likely value of $\omega$-that is, it is a one-dimensional problem. A least-squares solution would require the two basis functions (sin and cos) and locating a maximum in a three-dimensional space; similarly, a direct application of maximum entropy would require finding the mode of a threedimensional function.

The model, viewed as a differential equation, is a simpler expression than that above. It is simply

$$
\mathcal{M}(\alpha) \equiv\left[\mathrm{s}^{\prime \prime}+\alpha \mathrm{s}^{\prime}=0\right],
$$

where ' denotes time derivative. There is only one parameter and it appears linearly. The principle of maximum entropy is still used, but on $d_{k}=s_{k}+e_{k}$ instead of on $d_{k}=f\left(t_{k}\right)+e_{k}$ where $d_{k}$ is the data sequence, $e_{k}$ the presumed noise se- 
quence, and $s_{k}$ the output of the differential equation model; $f$ is the model function in the usual development. The two expressions for the data seem to be equivalent, but by taking second differences and using the fact that the differential equation may be written as the difference equation,

$$
\Delta^{2} \mathrm{~s}_{\mathrm{k}}+\alpha \mathrm{s}_{\mathrm{k}}=0,
$$

where $\Delta^{2}$ is the second difference, the noise sequence obeys the same difference equation $\Delta^{2} \mathrm{e}_{\mathrm{k}}+\alpha \mathrm{e}_{\mathrm{k}}=0$.

In this simple example, the maximum of the likelihood function occurs at a zero of the quadratic expression

$$
\sum_{k}\left(\Delta^{2} d_{k}+\alpha d_{k}\right)^{2} \text {. }
$$

Expanding the sums results in a quadric equation for $\alpha$ with coefficients that are correlations of the original time series. Since there are only a finite sequence of samples, the usual solution to the more general problem involving additional derivatives of $s$ and additional linear parameters is to apply Levinson's recursion for an autoregressive time series.

\section{CODEBOOK CONSTRUCTION}

Characterization of a natural system $\mathcal{N}$ from a causally generated sequence of observations-a time series, say-results in a model $\mathcal{M}$ for $\mathcal{N}$ by the process described above. From this model, one can then proceed to a simulation $\mathcal{S}$ wherein the causal entailment structure of $\mathcal{N}$ will most likely become superseded by the particular way the computer program specifying $\mathcal{S}$ is written; this structure is retained in $\mathcal{M}$, but lost in $S$. See Rosen [9, p. $182 \mathrm{ff}$.] for a discussion of these issues. The simulation will, however, allow comparisons between predictions generated by $\mathcal{S}$ with future behavior of $\mathcal{N}$ even though the complete semantic structure contained in decoding the inferential activities will have been lost-a touchstone to reality is retained even if one can no longer be assured of comparing entailments. What can be gained by making use of this restricted semantics?

Consider a differential equation with an arbitrary number of constant coefficients. Application of the above method will produce a particular set of values for these parameters at any given observation epoch. This set can be thought of as a vector and quantized in the usual manner to produce a codebook capturing the states of a system over time. A method of parsing the output of such a system is then dependent on the quantization step and the differential equation models that comprise the hypothesis set. When the behavior changes enough to leave a particular equivalence class as specified by an index on the hypothesis in question and the granularity of the quantization, a new behavior is identified and mapped onto a new codebook entry.

\section{Codebook as a Formal Model}

Consider constructing a codebook by vector quantization on $\{\alpha\}$, the set of parameters specifying a particular differential equation indexed from a set of likely candidates. The codebook entries, $\{c\}$, are to be viewed as a set of symbols that serve as an alphabet of individual behaviors of the natural system being formalized. The dynamics of the natural system is captured in a sequence (chronicle) of symbols that are seen as words in some formal language describing system behavior. A set of words generated from $\{c\}$ forms a semigroup. This view gives a structure to the dynamics which is decoded as a specific set of words. The dynamics inherits its structure from the semigroup structure. However, this semigroup structure is too general, so further restrictions are required. What is needed is something like "wellformed sentences," a grammar, in other words.
Inverse Modeling Relation

By "inverse" modeling, I mean the construction of an artificial, material, and hence, natural system based on the formal system abstracted from a set of other natural systems. This is, after all, what one wishes to accomplish by creating systems that behave as, or even are, organisms with the capability of independent and self-motivated behaviors. The hopes and wishes of so many seem to be to incorporate concepts of intelligence, consciousness, and life in artificial constructs; yet, apart from some clever metaphors and analogies, these ideas must await further development of theory as well as stand on the success of producing a first simple organism.

The theory of passing from formal systems to material systems, while closely related to theoretical engineering, is yet to be developed. After all, the theory of extracting formal models from natural systems-other than science embodied in various individual scientific disciplines-is a new endeavor. For example, the theory of abstracting formal systems from biological systems is certainly not in final form in spite of the brilliant work of Rosen and other mathematical biologists.

The conceptual relationship or material entailment of the construction process becomes $\left\{\mathcal{N}_{k}\right\} \rightarrow \mathcal{F} \rightarrow C$ where the individual $\mathcal{N}_{k}$ and $C$. (the construct) as well, are in world 1 , while $\mathcal{F}$ is in world 3 . I propose a constructive methodabstract at this stage- of obtaining $C$ by extending the codebook derived above. There are several ways to accomplish this, and probably many more will occur to the reader. For example, the codebook above can be veiwed as a Voronoi diagram, perhaps multidimensional. Thought of in this way as a geometric structure, one can imagine refining the Voronoi cells by assuming a different granularity of the decoding process-simply allow 16 bits for each parameter instead of 8. Extend the spatial domain covered by the cells. Augment the dimension of the parameter space. For example, let certain constants - such as frequency or decay ratebecome variables. Allow a parameter such as frequency to vary with time within a particular representation-thus turning a pure tone into a chirp or wail in an acoustic example. Of course these augmentations are suggestions for exploration; their utility as an augmented formal system describing a natural system must be determined by more measurements and hypothesis testing on, perhaps, a different set of differential equations.

Example Suppose we have a differential equation that is a model of a material system-say an harmonic oscillator consisting of a mass and a spring with perhaps internal damping. The formal system, derived from the simulation of this natural system, is the differential equation with its free parameters of frequency, damping constant, amplitude, phase, and so on. At one level of modeling, we may observe the behavior of the material system by making measurements on the position of the mass as a function of time-a chronicle, in Rosen's parlance [9]. The encoding process here is a simple time series of position measurements. Yet, with the correct model (differential equation), or one that is sufficiently close to correct, all the parameters necessary for the syntactic specification of the formal model may be determined from within an enlarged model by the usual activities of formal (mathematical) inference on a set of hypotheses using Bayes' rule and the principle of maximum entropy $[14,16]$ as indicated above.

It is not too hard to imagine extending the codebook obtained from the observed damped-oscillatory behavior to a vocoder that produces understandable speech. From initial observations of pure tones that possess attack and decay 
properties, the initial formal model is then used to observe speech. Close similarities are achieved by extending the codebook as described above and comparing the symbolic word sequences with the speech actually encoded. These extensions are thus guided by the natural system of speech production; of course, this process requires the creativity of the experimenter to stay within the set of simple differential equations and reasonable codebook extensions.

\section{An Amusing Deduction}

While the construct, $C$, is the embodiment of some formal system in the sense of material causation, it is not isomorphic to any formal system - certainly not the formal system $\mathcal{F}$ abstracted from $N$. Folklore concerning a robot surprising its maker is seen as a consequence of the inverse modeling relation that engenders $C$. By "surprise," I mean behaviors not arising as inferences within $\mathcal{F}$, otherwise we could "trivially" avoid surprises by turning the mathematical crank until all inferences were made (however, what if Gödel's theorem is applicable to $\mathcal{F}$, as it most likely is?). It is only in the highly unlikely situation that we have fully accounted for all possible decodings of $\mathcal{F}$ when constructing $C$ that we will get no surprises. The only way to minimize surprises is to make a class of formal models of $C$; call this class $\mathcal{F}^{*}$. If there is some largest member of $\mathcal{F}^{*}$, then there will be no surprises. If, however, $C$ is an organism, Rosen [9, pp. 244, ff.] has shown that there is no largest member of $\mathcal{F}^{*}$ with the consequence that $C$ will always contain surprises. Of course, this observation is entirely within our common-sense expectations of natural organisms. What is interesting is that this result is obtained by simple deduction within the framework of the modeling relation.

This argument may be turned around, retaining properties that Rosen has identified with organism, to show that a computer as currently conceived and realized by any given workstation or PC, or collection such entities, even connected by the Internet, cannot be an organism. Such concepts as intelligence and consciousness do not apply to assemblies of present-day computers other than by the trivial process of forming word analogies as in "artificial neural networks can be made to think since the human brain consists of neurons and humans think."

\section{SUMMARY}

I have presented a case for basing intelligent systems design on Rosen's modeling relation instead of the (to me, at least) more conceptually obscure and operationally difficult approach taken by researchers in applied semiotics. The construction of formal models that have "good" comparisons to certain natural systems was shown to be achievable by a method of Bayesian system identification that operates on the system structure responsible for producing observable behavior. For many natural systems, this structure is contained in the differential equations producing the behavior rather than on descriptions of functions that are solutions to those equations. The structures are seen as analogous to system semantics, while the behaviors themselves necessarily entangle system structure with environmental particulars, interesting only when abstracted into a larger model.

The framework of the modeling relation as developed by Rosen and embedded in Popper's three worlds, which incidentally retains the structure of semiosis, is certainly convenient in categorizing these ideas and procedures; it may even be necessary to avoid confusion. Most of the ideas presented above are neither new nor original. The only novelties are to seek system behavior by applying Bayesian methods directly to differential-equation hypotheses (this is often more practical than using basis functions), and constructing a codebook based on the parameter sets. This codebook may then be extended by certain formal operations, perhaps using methods of evolutionary programming. The idea to base a material construct on a direct decoding of a codebook instead of, or in addition to, verifying predictions in the natural system being modeled, seems powerful, yet needs further development. The entailments in the material construct are to be compared to behaviors in some, perhaps the same, natural system by further encoding. Does this give us intelligent behavior? Not yet, but I maintain that this procedure or something analogous to it is an essential step in constructing systems that actually exhibit intelligence.

\section{ACKNOWLEDGMENTS}

I would like to acknowledge the inspiration provided by the writings of E. T. Jaynes [1922-1998] whose clarity of thought and ability to create new and important ideas were only matched by his depth of perception and beauty of exposition.

This research was performed at Oak Ridge National Laboratory, managed by Lockheed Martin Energy Research, Inc., for the U.S. Department of Energy under Contract No. DEAC05-96OR22464.

\section{REFERENCES}

[1] Umberto Eco, Semiotics and the Philosophy of Language, Indiana University Press, Bloomington, 1984. [2] Alexander M. Meystel, "Intelligent Systems: A Semiotic Perspective," International Journal of Intelligent Control and Systems, 1(1), pp. 31-57, 1996.

[3] James S. Albus, "Outline for a Theory of Intelligence," IEEE Transactions on Systems, Man, and Cybernetics, 21(3), 1991.

[4] James S. Albus and Alexander M. Meystel, "A reference model architecture for design and implementation of intelligent control in large and complex systems," International Journal of Intelligent Control and Systems, 1(1), pp. 1530, 1996.

[5] Thomas Sebeok, Signs: An Introduction to Semiotics, University of Toronto Press, Toronto, 1994.

[6] The Sign of Three, edited by Umberto Eco and Thomas A. Sebeok, Indiana University Press, Bloomington, 1983.

[7] Karl R. Popper, Popper Selections, edited by David Miller, Princeton University Press, 1985.

[8] Robert Rosen, Anticipatory Systems, Pergamon Press, New York, 1985.

[9] Robert Rosen, Life Itself, Columbia University Press, New York, 1991.

[10] D. A. Pospelov, Situational Control, Nauka Publishers, Moscow, 1986.

[11] Thomas A. Goudge, The Thought of C.S. Peirce, Dover Publications, New York, 1950.

[12] R. T. Cox, "Probability, Frequency and Reasonable Expectation," American Journal of Physics, 17, pp. 1-13, 1946.

[13] E. T. Jaynes, Probability Theory-The Logic of Science, in press.

[14] E. T. Jaynes, "Information Theory and Statistical Mechanics," Physical Review, 106(4), pp. 620-30, 1956.

[15] G. Larry Bretthorst, Bayesian Spectrum Analysis and Parameter Estimation, Springer-Verlag, New York, 1988.

[16] E. T. Jaynes, "Bayesian Spectrum and Chirp Analysis," Maximum Entropy and Bayesian Spectral Analysis and Estimation Problems, C. Ray Smith and G. J. Erickson editors, D. Reidel, Dordrecht-Holland, pp. 1-37, 1987. 